L'exercice de la traduction a toujours joué un rôle important dans le ARTICULOS $\cdot 65-77$

développement du savoir humain. C'est pourquoi nous avons jugé intéressant d'étudier les traductions vers les langues française et espagnole des contes d'Edgar Allan Poe. Le but de cet article est l'étude de la cohésion temporelle dans une perspective cognitive dans trois contes d'Edgar Allan Poe : Berenice, The Oval Portrait, et The Masque of the Red Death, en partant du texte original anglais et en comparant les traductions vers les deux textes cibles. Nous aborderons donc le thème des marqueurs temporels, et plus concrètement, des marqueurs still, again et then qui nous ont semblé les plus intéressants dans notre optique traductrice. Nous partons de l'idée que ces marqueurs sont polysémiques et qu'ils ont un sens prototypique qui est temporel. À cette valeur centrale s'ajoutent d'autres valeurs que nous dénommerons périphériques (la focalisation, la conséquence, l'intensification). Cette caractéristique polysémique du marqueur induit fréquemment le traducteur à une interprétation erronée.

MOTS-CLÉs : traduction, analyse du discours, cohésion textuelle, marqueurs temporels.

\title{
Aspects discursifs dans les contes d'Edgar Allan
Poe et leurs traductions en français et en espagnol
}

Discursive aspects of Edgar Allan Poe's Tales and their translations in French and Spanish

It is known that the translation exercise has always been important in the development of the human knowledge. That is why we have considered interesting to analyse the French and Spanish translations of Edgar Allan Poe's Tales. The aim of this contribution is the study of temporal cohesion in a cognitive approach. We have chosen three Tales of Edar Allan Poe: Berenice, The Oval Portrait, and The Masque of the Red Death. In a first step we'll find the three markers still, again and then among the three Tales and in a second

Noelia Micó Romero

Universitat de València step we will compare the translations (French and Spanish ) of these markers with the original text. We think that these markers are polysemous and that they have a prototypic sense which is temporal and outlying senses like focalization, consequence or intensification. This polysemous characteristic provokes sometimes confusion among the translators.

KEY WORDS: translation, discourse analysis, textual cohesion, temporal markers. 


\section{INTRODUCTION}

Lobjectif de ce travail est l'analyse des mar66 queurs temporels ${ }^{1}$ suivants: still, again et then, dans les trois contes d'Edgar Allan Poe: Berenice (1835), The Masque of the red Death (I84I) et The Oval Portrait (1842) ainsi que leurs traductions vers la langue française (par Charles Baudelaire) et vers l'espagnol (par Julio Cortázar). Dans un premier temps, nous aborderons la notion de temporalité, dans un second temps, nous présenterons notre perspective d'analyse. En effet, nous nous situons dans le courant de la linguistique cognitive qui conçoit les mots polysémiques en tant que catégories radiales dans lesquelles nous pouvons distinguer un sens prototypique et des sens périphériques qui séloignent de la temporalité pour acquérir d'autres valeurs (notamment argumentatives). Finalement, nous présenterons les usages les plus intéressants des marqueurs still, again et then, que nous recueillons dans notre corpus d'exemples, pour pouvoir, de la sorte, analyser les différentes traductions vers le français et l'espagnol. De notre analyse, nous pouvons avancer que les traducteurs vers le français et l'espagnol ont parfois omis de traduire certains marqueurs. Notre analyse essaiera de comprendre le pourquoi de ces omissions.

\section{HYPOTHÈSE}

L'hypothèse que nous avançons est que les traducteurs, se trouvant parfois face à des cas ambigus en ce qui concerne les marqueurs temporels, objets de notre étude, décident dans certains cas de ne pas les traduire. En effet, ces marqueurs polysémiques acquièrent des valeurs

\footnotetext{
I Nous avons déjà traité les marqueurs temporels dans Micó Romero, N., 2002 y 2005.
}

que nous dénommerons périphériques (focalisation, argumentation) et qui s'éloignent de leur valeur temporelle prototypique. Pour éviter toute confusion, une option facile est d'omettre ces marqueurs dans la traduction.

\section{CADRE THÉORIQUE DE L'ÉTUDE}

\section{Notion de temporalité}

Notre étude a pour base des contes ou récits courts. D'une part nous suivons la linguistique cognitive pour expliquer les notions de prototypicalité et de périphérique et d'autre part, nous nous situons dans une perspective textuelle et discursive car les traducteurs se confrontent à des textes originaux qu'ils doivent interpréter correctement Dans ce sens, un texte, pour qu'il soit considéré comme tel, doit constituer un tout homogène. Il a donc besoin non seulement de marques de cohésion comme la référence, la deixis, la connexion et la temporalité, mais aussi de marques de cohérence comme la progression thématique. De tous les aspects cités, celui qui nous intéresse le plus est celui de la temporalité, car cette notion joue un rôle important dans la localisation interne $\mathrm{du}$ discours ainsi que dans la contextualisation. D'un côté, nous entendons par localisation interne, la notion de temps qui va garantir un agencement correct des énoncés les uns par rapport aux autres sur la ligne du temps. D’un autre côté, un des principaux axes sur lequel se construit le sens sont les contextualisations spatiales et temporelles, dans la mesure où elles représentent un continuum sur le quel viennent se situer les événements. Dans ce sens, les marqueurs temporels jouent un rôle important car ils garantissent l'agencement correct des énoncés dans un récit.

La temporalité se traduit dans le texte à l'aide des temps verbaux, des circonstants temporels 
(i.e. en I9I8), des déictiques (i.e. aujourd'bui, demain, etc.), des organisateurs du discours (i.e. en premier lieu, en deuxième lieu, etc.), de la syntaxe temporelle, mais surtout à travers des marqueurs temporels (i.e. encore, alors), objets de notre étude.

\section{La notion de catégorie radiale de la linguistique cognitive ${ }^{2}$}

Comme nous l'avons déjà dit supra, la linguistique cognitive peut nous fournir le cadre théorique qui explique les différentes valeurs de ces marqueurs. En effet, la linguistique cognitive considère les mots polysémiques en tant que catégories radiales. C'est pourquoi, les marqueurs temporels possèdent une structure interne qui contient une constellation de sens. Ces valeurs se retrouveront dans une certaine mesure dans les traductions. Par exemple, le marqueur temporel anglais then, peut être considéré comme polysémique. Autrement dit, il possède une structure radiale qui comprend des usages prototypiques comme celui de succession temporelle (les événements se succèdent les uns après les autres) que nous appellerons valeurs centrales et d'autres plus périphériques, ou moins prototypiques comme la conséquence.

\section{MATÉRIEL UTILISÉ}

Nous avons choisi trois contes d'Edgar Allan Poe car nous pensons que c'est un auteur très important de la littérature anglophone du XIX siècle. Il a été un des grands écrivains de la nouvelle et s'inscrit dans le courant romantique américain. Il a eu une grande

\footnotetext{
2 Nous ne pouvons pas nous attarder sur cette notion clé de la sémantique cognitive, et nous renvoyons les lecteurs à Cuenca, M. J. \& Hilferty, J. (I999), Kleiber, G. (1990) , et Langacker, R-W. (1987).
}

influence non seulement sur les écrivains américains tels que Mark Twain, Herman Melville, Ambrose Bierce, Ray Bradbury, et Howard Phillips Lovecraft, mais aussi sur delaire - qui a traduit cinq ouvres de PoeVictor Hugo, le comte de Lautréamont, Paul Verlaine, Stéphane Marallarmé, Arthur Rimbaud, Paul Valéry, Marcel Proust, Guy de Maupassant et Jules Verne. En Espagne, il a eu une grande répercussion sur Pío Baroja et Vicente Blasco Ibáñez par exemple. En Amérique Latine, il a reçu une grande admiration de la part d'auteurs tels que Rubén Darío, Horacio Quiroga, Jorge Luis Borges y Julio Cortázar qui a traduit aussi quelques œuvres de Poe. Celui-ci eut aussi beaucoup de succès auprès des écrivains russes, suédois et grecs.

Les traducteurs des contes que nous avons choisis pour notre travail sont Charles Baudelaire pour la version française et Julio Cortázar pour la version espagnole. Les contes que nous avons sélectionnés sont les suivants : Berenice (1835), The Masque of the Red Death (I84I) et The Oval Portrait (1842), ainsi que leurs traductions vers le français et l'espagnol. La fréquence d'apparition des marqueurs then, still et again a déterminé le choix de ces œuvres. De même ces marqueurs temporels ont été sélectionnés car nous avons observé qu'ils présentaient des cas d'anomalies dans le sens où ils se déviaient de leur valeur centrale, la temporalité, pour acquérir de nouvelles valeurs plus périphériques.

Pour notre commentaire, nous avons choisi les exemples les plus significatifs. Ceux-ci apparaittront toujours dans le même ordre: d'abord en anglais puisque c'est la langue de départ, ensuite en français et finalement en espagnol. 
Tableau I

\begin{tabular}{|c|c|c|c|}
\hline \multicolumn{2}{|r|}{ Traductions } & \multicolumn{2}{|c|}{ Valeurs de stilla } \\
\hline & & Temporel indiquant la durée & Intensifieur \\
\hline \multirow{2}{*}{ FRANÇAIS $^{b}$} & Encore & $B^{c} 23$ & В 25, В 25, В 25, \\
\hline & Toujours & B 24, B 24, B 24, & \\
\hline Total & 7 & 4 & 3 \\
\hline \multirow{3}{*}{ ESPAGNOL $^{\mathrm{d}}$} & Aún & В 23, В 25, В 25, В 25, & \\
\hline & Seguir + adjectif & & B 24, B 24, \\
\hline & Mantener + substantif & & B 24, \\
\hline Total & 7 & 4 & 3 \\
\hline
\end{tabular}

a Dans tous les tableaux, les valeurs des marqueurs en anglais sont tirées du dictionnaire unilingue anglais (20II) Collins English Dictionary. London : Harper Collins Publishers.

b Dans tous les tableaux, les valeurs des marqueurs en français sont tirées de Franckel, J. J. (I989). Étude de quelques marqueurs aspectuels du français. GinebraParis: Librairie Droz.

c Dans tous les tableaux, les abréviations MR, B et PO correspondent respectivement aux trois titres d'Edgar Allan Poe que nous allons étudier dans cet article, à savoir The Masque of the red Death, Berenice et The Oval Portrait. Le chiffre qui suit correspond au numéro de l'exemple dans notre corpus.

d Dans tous les tableaux, les valeurs des marqueurs en espagnol sont tirées de Bosque, I. \& Demonte, V. (200o [1999]). Gramática descriptiva de la lengua española, vol I: Sintaxis básica de las clases de palabras. Madrid: Espasa Calpe.

\section{LE MARQUEUR STILL}

\section{Tableau récapitulatif des valeurs de still dans notre corpus [Tableau I]}

\section{Étude de cas : le marqueur still}

Dans le corpus d'exemples que nous avons recueilli, le marqueur still est temporel et exprime la durée. Dans la version française, ce marqueur est traduit ou bien par encore ou bien par toujours (ayant pour valeur la permanence dans le temps). Dans la version espagnole, il est traduit par le marqueur aún qui exprime aussi la durée ou bien, comme c'est le cas de l'exemple choisi, par la périphrase verbale (seguir + adjectif) ou encore par le sémantème verbal (mantener + substantif). En français, nous avons aussi trouvé trois exemples où le marqueur fonctionne comme marqueur d'intensité du parti- cipe tandis qu'en anglais, il porte sur le temps fini/conjugué à la forme personnelle.

r. $\mathrm{a}^{3}$. And the evening closed in upon me thus-and then the darkness came, and tarried, and went — and the day again dawned — and the mists of a second night were now gathering around —and still I sat motionless in that solitary room; and still I sat buried in meditation, and still the phantasme of the teeth maintained its terrible ascendancy as, with the most vivid hideous distinctness, it floated about amid the changing lights and shadows of the chamber. (B 24, p. 6)

3 Le chiffre correspond au numéro de cet exemple dans notre article. Les lettre a, b et c correspondent respectivement à la version anglaise, la version française et la version espagnole.

${ }_{4}$ Comme nous avons dit supra, les abréviations MR, $\mathrm{B}$ et $\mathrm{PO}$ correspondent respectivement aux trois titres 
TableaU 2

\begin{tabular}{|c|c|c|}
\hline \multicolumn{2}{|r|}{ Traductions } & Valeurs de again \\
\hline & & $\begin{array}{c}\text { Temporel indiquant } \\
\text { l'itération }\end{array}$ \\
\hline \multirow{4}{*}{$\begin{array}{l}\text { FRAN- } \\
\text { ÇAIS }\end{array}$} & De nouveau & MR I7, PO I9 \\
\hline & Encore & PO 20 \\
\hline & Nouveau & $\mathrm{PO} 2 \mathrm{I}$ \\
\hline & Sans traduction & MR I8, PO 22 \\
\hline Total & 6 & 6 \\
\hline \multirow{5}{*}{$\begin{array}{l}\text { EsPA- } \\
\text { GNOL }\end{array}$} & De nuevo & PO I9, PO 22 \\
\hline & Otra vez & MR I7 \\
\hline & Aún & PO 20 \\
\hline & Nuevo & PO 2I \\
\hline & Sans traduction & MR I8 \\
\hline Total & 6 & 6 \\
\hline
\end{tabular}

r.b. Et le soir descendit ainsi sur moi, — et les ténèbres vinrent, s'installèrent, et puis s'en allèrent, - et un jour nouveau parut, - et les brumes d'une seconde nuit s'amoncelèrent autour de moi, - et toujours je restais immobile dans cette chambre solitaire, - toujours assis, toujours enseveli dans ma méditation, — et toujours le fantôme des dents maintenait son influence terrible, au point qu'avec la plus vivante et la plus hideuse netteté il flottait çà et là à travers la lumière et les ombres changeantes de la chambre. (B 24, p. 8).

I.c. Y la tarde cayó sobre mí, y vino la oscuridad, duró y se fue, y amaneció el nuevo día, y las brumas de una segunda noche se acumularon y yo seguía inmóvil, sentado en

d'Edgar Allan Poe, à savoir The Masque of the red Death, Berenice et The Oval Portrait. Le chiffre qui suit correspond au numéro de l'exemple dans notre corpus. Et finalement, la page du conte d'où provient cet exemple. aquel aposento solitario; y segui sumido en la meditación, y el fantasma de los dientes mantenía su terrible ascendiente como si, con la claridad más viva y más espantosa, flotara entre las cambiantes luces y sombras del recinto. (B 24, p.6)

Maintenant voyons le cas où le marqueur still indique l'intensité et la continuité.

2.a. (...) of a disfigured body enshrouded, yet still breathing, still palpitating, still alive! (B 22, p. 7)

2.b. (...) d'un corps défiguré, dépouillé de son linceul, mais respirant encore, - palpitant encore, - encore vivant ! (B 22, p. II)

2.c. (...) y su voz cobró un tono espeluznante, nítido, cuando me habló, susurrando, de una tumba violada, de un cadáver desfigurado, sin mortaja y que aún respiraba, aún palpitaba, aún vivía. ( $\mathrm{B}_{22}, \mathrm{p} .7$ )

Nous pouvons observer que le marqueur still se dévie de sa valeur centrale (temporelle) et acquiert une valeur périphérique (intensifieur) : le corps respire, palpite et finalement vit encore. Il s'agit d'une intensification en gradation ascendante.

Le marqueur still est le moins enrichissant, car il ne présente pas de cas ambigus. Il peut indiquer la valeur temporelle de durée ou comme intensifieur d'un adjectif et il est traduit dans toutes les occurrences trouvées dans notre corpus, par encore et toujours pour la version française et par aún (duratif), par Seguir + adjectif et par Mantener + substantif pour la version espagnole.

\section{LE MARQUEUR $A G A I N$}

\section{Tableau récapitulatif des valeurs de again dans notre corpus [Tableau 2]}

\section{Étude de cas du marqueur again}


Dans les trois textes étudiés, nous avons relevé six exemples qui contiennent le marqueur again, et tous indiquent l'itération. En ce qui concerne les traductions, nous avons pu observer que pour la version française, on a traduit again par de nouveau, par encore (indiquant tous deux l'itération) ou par l'adjectif nouveau qui exprime aussi la répétition. Dans la traduction espagnole, le marqueur again a été traduit le plus souvent par de nuevo ou par otra vez qui indiquent aussi l'itération.

3.a. In a very few moments I again looked fixedly at the painting. (PO, p. 2)

3.b. Au bout de quelques instants, je regardai de nouveau la peinture fixement. (PO, p. 2)

3.c. Al cabo de algunos momentos, miré de nuevo el lienzo fijamente. (PO, p. 2)

Dans l'exemple qui suit, le marqueur again a été restitué par le marqueur aún qui, dans aucun cas ne peut exprimer l'itération. En effet, il indique plutôt la durée dans le temps.

4.a. And when many weeks bad passed, and but little remained to do, save one brush upon the mouth and one tint upon the eye, the spirit of the lady again flickered up as the flame within the socket of the lamp. (PO I7, p. 3)

4.b. Et quand bien des semaines furent passées et qu'il ne restait plus que peu de chose à faire, rien qu'une touche sur la bouche et un glacis sur l'oeil, l'esprit de la dame palpita encore comme la flamme dans le bec d'une lampe. (PO I7, p. 4)

4.c. Y cuando muchas semanas hubieron transcurrido, y no restaba por hacer más que una cosa muy pequeña, sólo dar un toque sobre la boca y otro sobre los ojos, el alma de la dama palpitó aún, como la llama de una lámpara que está próxima a extinguirse.( PO I7, p.3)
Nous proposons la traduction suivante :

Y cuando muchas semanas hubieron transcurrido, y no restaba por hacer más que una cosa muy pequeña, sólo dar un toque sobre la boca y otro sobre los ojos, el alma de la dama palpitó una vez más, como la llama de una lámpara que está próxima a extinguirse.

Une conjecture qui pourrait être faite serait que Julio Cortázar a élaboré sa traduction à partir du texte français et qu'il aurait traduit par mégarde ce marqueur encore par aún (qui serait la valeur prototypique de ce marqueur, à savoir la valeur temporelle de durée) plutôt que par una vez más (valeur plus périphérique).

En outre, dans la traduction, ce marqueur again a été omis à deux reprises contre une seule omission pour le texte espagnol.

5.a. He made his way uninterruptedly, but with the same solemn and measured step which had distinguished him from the first, through the blue chamber to the purple through the purple to the green - through the green to the orange - through this again to the white - and even thence to the violet, ere a decided movement had been made to arrest him.( MR I8. p5)

5.b. Il continua sa route sans interruption, de ce même pas solennel et mesuré qui l'avait tout d'abord caractérisé, de la chambre bleue à la chambre pourpre, - de la chambre pourpre à la chambre verte, - de la verte à l'orange, - de celle-ci [à nouveau $]^{5}$ à la blanche, - et de celle-là à la violette, avant qu'on eût fait un mouvement décisif pour l'arrêter. (MR I8, p. 6)

5.c. Siguió andando ininterrumpidamente pero con el mismo y solemne paso que desde el principio lo había distinguido. Y de la cámara azul pasó la púrpura, de la púrpura

Les mots entre crochets sont à nous. 
a la verde, de la verde a la anaranjada, desde ésta $[\text { otra vez }]^{6}$ a la blanca y de allí, a la violeta antes de que nadie se hubiera decidido a detenerlo.( MR I8, p. 5)

On pourrait penser que les traducteurs français et espagnol ont décidé d'omettre ce marqueur, ils n'ont probablement pas su déceler que dans le texte original, l'auteur fait retomber l'itération su le fait de changer de chambre. Ils ont interprété que cette répétition concernait la couleur de la chambre. Or la chambre de couleur blanche n' a pas encore été mentionnée, et les traducteurs on décidé de ne pas traduire le marqueur.

Dans l'exemple 6, le traducteur français a décidé d'omettre le marqueur again qui indique l'itération et a choisi de restituer ce marqueur par deux propositions juxtaposées. En revanche, le traducteur espagnol a bien traduit again par de nuevo qui indique également l'itération.

6.a.I found myself sitting in the library, and again sitting there alone. ( $\mathrm{B} 22, \mathrm{p} .7)$

6.b. Je me retrouvai dans la bibliothèque ; j'étais assis, jétais seul. (B 22, p. Iо)

6.c. Me encontré sentado en la biblioteca y de nuevo solo. (B 22, p. 7)

Pour la version française, nous proposons la traduction suivante : "Je me retrouvai assis dans la bibliothèque ; et une fois de plus, j'étais seul ».

On observe donc que le marqueur again pose certains problèmes aux traducteurs. Dans notre corpus d'exemples, nous n'avons trouvé qu'une seule valeur, celle d'itération. Il s'est traduit vers le français tantôt par de nouveau, tantôt par encore. En espagnol, il a été traduit par luego ou par entonces.

6 Les mots entre crochets sont à nous.
LE MARQUEUR THEN

\section{Tableau récapitulatif des valeurs $d u$} marqueur then dans notre corpus [tableau 3]

\section{Étude de cas du marqueur then}

Voyons le premier exemple qui traduit la succession temporelle.

7.a. There were sharp pains, and sudden dizziness, and then profuse bleeding at the pores, with dissolution. (MR I, p. I)

7.b. C'étaient des douleurs aiguës, un vertige soudain, et puis un suintement abondant par les pores, et la dissolution de l'être. (MR I, p. I)

7.c. Comenzaba con agudos dolores, un vértigo repentino, y luego los poros sangraban y sobrevenía la muerte. (MR I, p. I)

Dans cet exemple, nous pouvons observer que la succession temporelle est traduite en français par puis et en espagnol par luego. Voyons quel est l'éventail de valeurs que ces marqueurs peuvent exprimer en français et en espagnol.

\section{Valeurs de puis et de luego [tableau 4 ]}

Puis et luego possèdent les mêmes valeurs de succession dans le temps et dans l'espace ainsi que celle d'addition (dans le sens où on introduit un nouvel argument). Comme nous pouvons observer dans le tableau ci-dessus, puis et luego renferment les mêmes valeurs sauf la troisième où en français puis peut introduire le dernier terme d'une énumération et une cause ajoutée tandis que pues et luego peuvent introduire une conséquence, valeur que puis ne contient pas. Nous observons donc une asymétrie entre ces trois marqueurs. 
TABLEAU 3

\begin{tabular}{|c|c|c|c|c|c|c|}
\hline & \multirow[b]{2}{*}{ Traductions } & \multicolumn{5}{|c|}{ Valeurs de then } \\
\hline & & $\begin{array}{c}\text { Temporel } \\
\text { anaphorique }\end{array}$ & $\begin{array}{l}\text { Succession tem- } \\
\text { porelle }\end{array}$ & Consécutif & Focalisateur & $\begin{array}{l}\text { transphras- } \\
\text { tique }\end{array}$ \\
\hline \multirow{6}{*}{$\begin{array}{l}\text { FR A N - } \\
\text { ÇAIS }\end{array}$} & Puis & & MR I, MR 2, MR 5 & & MR 8 & \\
\hline & Alors & MR 3, MR 4, MR 6 & & MR 7 , B II & PO I6 & \\
\hline & Tout à l'heure & B Iо & & & & \\
\hline & Donc & & & & & B 13 \\
\hline & Enfin & & $\mathrm{B}$ I4 & & & \\
\hline & Sin traducción & В 9, В I2 & PO 15 & & & \\
\hline Total & I6 & 6 & 5 & 2 & 2 & $\mathrm{I}$ \\
\hline \multirow{7}{*}{$\begin{array}{l}\text { EsPA- } \\
\text { GNOL }\end{array}$} & Pues & & & & & $\mathrm{B}$ I3 \\
\hline & Luego & & MR I & & & \\
\hline & Entonces & MR 4, MR 6 & & B II & MR 8, PO I6 & \\
\hline & Digo & B 9 & & & & \\
\hline & Un momento antes & B IO & & & & \\
\hline & Mas & & MR 2 & & & \\
\hline & Sin traducción & MR 3, B I2 & B I4, MR 5, PO I5 & MR 7 & & \\
\hline Total & I6 & 6 & 5 & 2 & 2 & I \\
\hline
\end{tabular}

\section{TableaU 4}

\begin{tabular}{|c|l|l|}
\hline & \multicolumn{1}{|c|}{ Puis } & \multicolumn{1}{|c|}{ Luego } \\
\hline I & Indique la succession temporelle (ensuite) & \multicolumn{1}{|c|}{} \\
\hline 2 & Indique la succession dans l'espace (après, después) & \multicolumn{2}{|c|}{} \\
\hline 3 & $\begin{array}{l}\text { Et puis introduit le dernier terme dans une énumération. } \\
\text { Ex.: on entendit deux coups, et puis plus rien } \\
\text { Et puis introduit aussi une nouvelle cause explicative } \\
\text { Ex.: Et puis, ça ne me regarde pas }\end{array}$ & $\begin{array}{l}\text { Introduit une conséquence: } \\
\text { Ex.: Pienso, luego existo } \\
\text { ?? Je pense puis je suis/ } \\
\text { je pense donc je suis }\end{array}$ \\
\hline 4 & Introduit un nouvel argument ou élément (d'ailleurs, en outre) \\
\hline
\end{tabular}

Comme le montre l'exemple 7 , les traductions $\mathrm{du}$ marqueur then par puis et luego reprennent donc les valeurs de succession temporelle de ce marqueur.

L'exemple numéro 8 nous laisse un peu perplexes. En effet, le marqueur then, combiné avec la conjonction de coordination and indique normalement une focalisation, mais nous croyons que dans cet exemple, en plus d'une focalisation du moment où sonne l'horloge, l'auteur a voulu exprimer la succession des événements, c'està-dire l'itération du tintement de l'horloge qui se produit toutes les heures. Pour la traduction française, nous aurions préféré que le traducteur reste fidèle à la valeur de focalisation en traduisant and then par et à ce moment-là. 


\section{Tableau 5}

Mas

I. Le marqueur mas unit deux éléments qui dénotent que la notion exprimée par le deuxième s'oppose à la notion exprimée par le premier, sans être incompatible avec elle :

Ex.: Quique solía decir que eran manifestaciones de un sentimiento atávico; mas para Quique todo eran manifestaciones atávicas. ${ }^{\mathrm{e}}$

2. Ce marqueur unit deux éléments qui dénotent que la notion affirmée et exprimée dans la deuxième partie de la phrase s'oppose à la négation exprimée dans la première partie de la phrase, avec laquelle elle est incompatible.

Ex.: No tuvo inmediata respuesta de Suero de Quiñones, mas de otro caballero.

e Cet exemple est issu de Seco, M. (I999). Diccionario del español actual, Madrid: Grupo Santillana de ediciones, S.A., pp. $2982-2983$.

8.a. But when the echoes had fully ceased, a light laughter at once pervaded the assembly; the musicians looked at each other and smiled as if at their own nervousness and folly, and made whispering vows, each to the other, that the next chiming of the clock should produce in them no similar emotion; and then, after the lapse of sixty minutes, (which embrace three thousand and six hundred seconds of the Time that flies,) there came yet another chiming of the clock, and then were the same disconcert and tremulousness and meditation as before. (MR 2, p.3)

8.b. Mais, quand l'écho s'était tout à fait évanoui, une légère hilarité circulait par toute l'assemblée ; les musiciens s'entre-regardaient et souriaient de leurs nerfs et de leur folie, et se juraient tout bas, les uns aux autres, que la prochaine sonnerie ne produirait pas en eux la même émotion ; et puis, après la fuite des soixante minutes qui comprennent les trois mille six cents secondes de l'heure disparue, arrivait une nouvelle sonnerie de la fatale horloge, et c'était le même trouble, le même frisson, les mêmes rêveries.(MR 2, p. 3)

8.c. Pero apenas los ecos cesaban del todo, livianas risas nacían en la asamblea; los músicos se miraban entre sí, como sonriendo de su insensata nerviosidad, mientras se prometían en voz baja que el siguiente tañido del reloj no provocaría en ellos una emoción semejante. Mas, al cabo de sesenta y tres mil seiscientos segundos del Tiempo que huye, el reloj daba otra vez la hora, y otra vez nacían el desconcierto, el temblor y la meditación. (MR 2, p.3)

La traduction espagnole nous présente une traduction de then par mas.

Voyons maintenant l'ensemble des valeurs du marqueur mas : [tableau 5]

Dans l'exemple (8.c.), le traducteur introduit de manière consciente une nouvelle nuance d'opposition qui ne se trouve pas dans le texte original ni dans la traduction française. Ce marqueur mas en position initiale annonce la tragédie. Le narrateur déduit cette opposition des événements-mêmes : opposition entre la hilarité de l'assemblée et les sourires entre les musiciens et les coups de la fatale horloge qui sont une prémonition du malheur qui est à venir.

Lorsque le marqueur then a une valeur anaphorique, il se traduit normalement par alors/à ce moment-là en français et par entonces / en ese momento en espagnol.

9.a. And the revel went whirlingly on, until at length there commenced the sounding of 
Tableau 6

\begin{tabular}{|l|l|l|}
\hline \multirow{2}{*}{ I. } & \multicolumn{1}{|c|}{ Alors } & \multicolumn{1}{|c|}{$\begin{array}{l}\text { Valeur temporelle anaphorique: } \\
\text { où le marqueur reprend le temps de référence établit dans la proposition antérieure. }\end{array}$} \\
\cline { 2 - 3 } & $\begin{array}{l}\text { Ex.: Je vivais à Madrid quand j’avais cinq ans. Je } \\
\text { nétais qu'un enfant alors. }\end{array}$ & $\begin{array}{l}\text { Ex.: Vivía en Madrid cuando tenía cinco años. Yo no } \\
\text { era más que un crío entonces. }\end{array}$ \\
\hline \multirow{2}{*}{2.} & Valeur consécutive & Ex.: Se marchó. Entonces ya no hablemos de ello. \\
\cline { 2 - 3 } & Ex.: Il est parti. Alors n'en parlons plus. & \\
\hline 3 & Tour de parole & $\varnothing$ \\
\cline { 2 - 3 } & Ex: Alors, ça va? & \multicolumn{2}{|l}{} \\
\hline
\end{tabular}

f Nous ne rendons pas compte ici de toutes les valeurs en tant que marqueurs conversationnels, car ils ne sont pas l'objet de notre étude et n'apparaissent donc pas dans notre corpus d'exemples.

midnight upon the clock. And then the music ceased, as I have told; and the evolutions of the waltzers were quieted; and there was an uneasy cessation of all things as before. (MR 4, p. 4)

9.b. Et la fête tourbillonnait toujours lorsque s'éleva enfin le son de minuit de l'horloge. Alors, comme je l'ai dit, la musique s'arrêta ; le tournoiement des valseurs fut suspendu ; il se fit partout, comme naguère, une anxieuse immobilité. (MR 4. P4)

9.c. Continuaba la fiesta en su torbellino hasta el momento en que comenzaron a oírse los tañidos del reloj anunciando la medianoche. Calló entonces la música, como ya he dicho, $\mathrm{y}$ las evoluciones de los que bailaban se interrumpieron; y como antes, se produjo en todo una cesación angustiosa. (MR 4, p. 4)

Dans cet exemple (9), le marqueur temporel est anaphorique et reprend une référence temporelle antérieure (cfr. midnight / minuit/ medianoche). Voyons si cet usage anaphorique du marqueur en français et en espagnol sont repris dans leurs valeurs en langue.

\section{Valeurs en langue de alors 7 y de entonces ${ }^{8}$ [tableau 6]}

De toutes les valeurs en langue des marqueurs alors et entonces que nous avons relevées, nous pouvons souligner que tous les deux possèdent la valeur temporelle anaphorique et la valeur consécutive. Par conséquent, nous pouvons affirmer que les traductions alors et entonces reprennent la valeur temporelle anaphorique.

En ce qui concerne l'exemple suivant, le marqueur then est anaphorique mais dans le discours, c'est-à-dire qu'il n'est pas temporel anaphorique, il ne renvoie pas à un temps antérieur dans le sens de "à ce moment-là », il fait plutôt référence à une partie du discours que lon reprend après une digression.

7 Les valeurs des marqueurs en français sont tirées de Franckel, J. J. (1989). Étude de quelques marqueurs aspectuels du français. GinebraParis: Librairie Droz.

8 Les valeurs des marqueurs en espagnol sont tirées de Bosque, I. \& Demonte, V. (2000 [I999]). Gramática descriptiva de la lengua española. Vol I: Sintaxis básica de las clases de palabras. Madrid: Espasa Calpe. 
Io.a. In the meantime my own disease - for I have been told that I should call it by no other appellation - my own disease, then, grew rapidly upon me, and assumed finally a monomaniac character of a novel and extraordinary (B 9, p.3)

Io.b. En même temps, mon propre mal, car on m'a dit que je ne pouvais pas l'appeler d'un autre nom, - mon propre mal grandissait rapidement, et, ses symptômes s'aggravant par un usage immodéré de l'opium, il prit finalement le caractère d'une monomanie d'une forme nouvelle et extraordinaire. (B 9, p. 3)

Io.c. Entretanto, mi propia enfermedad - pues me han dicho que no debo darle otro nombre, mi propia enfermedad, digo, crecía rápidamente, asumiendo, por último, un carácter monomaniaco de una especie nueva y extraordinaria, que ganaba cada vez más vigor y, al fin, obtuvo sobre mí un incomprensible ascendiente. (B 9, p.3)

Dans ce cas, le marqueur then nous dévoile l'attitude du narrateur. Celui-ci est conscient qu'il répète le mot disease (dans l'exemple roa) et après une digression explicative (on lui a dit qu'il ne pouvait pas l'appeler autrement), il se sent obligé de répéter ce mot pour maintenir la fonction conative, pour que le lecteur ne se perde pas. Il est intéressant de constater que dans la traduction française, on a omis ce marqueur et que dans la version espagnole, ce marqueur a été traduit par digo qui est beaucoup plus transparent que le marqueur then en anglais. Dans le cas de l'espagnol, le narrateur a choisi d'être plus explicite.

Nous voulons souligner un autre exemple curieux où le marqueur then à valeur anaphorique temporelle a été traduit par tout à l'beure en français et par un momento antes en espagnol.
II.a. I saw them now even more unequivocally than I beheld them then. (B Iо, p. 6)

Ir.b. Je les vis même alors plus distinctement que je ne les avais vues tout à l'heure. (B Iо, p. 7)

II.c. Los vi entonces con más claridad que un momento antes. (B Iо, p. 6).

Nous pensons qu'une traduction plus générique aurait été plus adéquate (...que je ne les avais vues auparavant / avant, pour la version française et tout simplement antes pour la version espagnole).

Après avoir étudié les valeurs prototypiques ou centrales du marqueur then, c'est-à-dire ses valeurs temporelles, poursuivons avec les valeurs plus périphériques comme la valeur consécutive.

D'après notre corpus d'exemples, la valeur consécutive du marqueur then a été restituée par alors et entonces en français et espagnol respectivement. Voyons un exemple où ce marqueur consécutif n'a pas été traduit dans la version espagnole.

I2.a. There was a sharp cry — and the dagger dropped gleaming upon the sable carpet, upon which, instantly afterwards, fell prostrate in death the Prince Prospero. Then, summoning the wild courage of despair, a throng of the revellers at once threw themselves into the black apartment, (...) (MR 7, p. 6)

I2.b. Un cri aigu partit, — et le poignard glissa avec un éclair sur le tapis funèbre où le prince Prospero tombait mort une seconde après. Alors, invoquant le courage violent du désespoir, une foule de masques se précipita à la fois dans la chambre noire ; (..) (MR 7, p. 7)

I2.c. Oyese un agudo grito, mientras el puñal caía resplandeciente sobre la negra alfombra, y el príncipe Próspero se desplomaba muerto. Poseídos por el terrible coraje 
de la desesperación, numerosas máscaras se lanzaron al aposento negro; (...) ( MR 7, p. 5)

Cette omission provoque une perte du point 76 de vue de la cohésion du texte dans la langue espagnole. Nous pensons que la polysémie de ce marqueur qui indique dans cet exemple la conséquence, valeur périphérique et non prototypique de ce marqueur a mis dans le doute le traducteur qui a préféré ne pas traduire ce marqueur.

D'après notre grille récapitulative, le marqueur then peut aussi acquérir une autre valeur périphérique comme la focalisation :

I3.a. Oh! Naiad among its fountains! — and then - then all is mystery and terror, and a tale which should not be told. (в 8, p. 2)

I3.b. ! Oh ! naïade parmi ses fontaines ! Et puis, - et puis tout est mystère et terreur, une histoire qui ne veut pas être racontée. (в 8, p.3)

I3.c. ¡Oh náyade entre sus fuentes! Y entonces, entonces todo es misterio y terror, y una historia que no debe ser relatada. (B 8, p. 2)

En anglais, la combinaison du marqueur then avec la conjonction de coordination and met l'accent sur le fait ou l'événement qui suit. Cette focalisation est parfaitement restituée en français par la combinaison et puis, focalisation qui se voit renforcée par la répétition de et puis. En espagnol, nous retrouvons le même procédé, la répétition de $y$ entonces.

La dernière valeur que reprend notre grille est la valeur transphrastique du marqueur Then.

I4.a. Why then, as I perused them, did the hairs of my head erect themselves on end, and the blood of my body become congealed within my veins? (B I3, p. 7.)

I4.b. D'où vient donc qu'en les lisant mes cheveux se dressèrent sur ma tête et que mon sang se glaça dans mes veines? (B I3, p. Io)
I4.c. ¿Por qué, pues, al leerlas se me erizaron los cabellos y la sangre se congeló en mis venas? (B I3, p. 7)

Dans cet exemple, le marqueur then trahit l'attitude de l'énonciateur et exprime l'incrédulité ou la surprise causée par les éléments antérieurs ou par ce qui s'observe par la suite.

Le marqueur then est polysémique et cette caractéristique peut engendrer des cas ambigus. C'est pourquoi nous pensons que le marqueur then appartient à une catégorie radiale qui contient un noyau central temporel. Il peut en effet indiquer que les événements se succèdent les uns aux autres, il peut reprendre une référence temporelle qui est apparue antérieurement dans le texte. À ces valeurs nucléaires s'en ajoutent d'autres que nous avons appelées périphériques (la focalisation, la conséquence et le cas transphrastique) parce qu'elles s'éloignent du noyau central qui est temporel.

\section{CONCLUSION}

Pour terminer, nous devons souligner qu'en raison de leur caractère polysémique qui provoque des situations ambigües, de nombreux marqueurs n'ont été traduits ni en français ni en espagnol (Io exemples pour tous les marqueurs confondus), laissant un vide de sens. Cela signifie que la facette polysémique des marqueurs temporels provoque une certaine confusion chez le traducteur. Cette confusion pousse le traducteur à omettre le marqueur dans sa traduction pour éviter de commettre une erreur. Mais, en ce qui nous concerne, nous pensons que ces marqueurs temporels ne doivent pas être ignorés parce qu'ils constituent des éléments fondamentaux pour la cohésion globale du texte.

C'est pourquoi, il est important de continuer les recherches sur les marqueurs en général et 
plus concrètement sur les marqueurs temporels afin de pouvoir observer comment ils se comportent dans d'autres types de discours.

RECIBIDO EN ENERO DE 2012 ACEPTADO EN FEBRERO DE 2OI2 VERSIÓN FINAL DE MARZO DE 2012

\section{RÉFÉRENCES BIBLIOGRAPHIQUES}

AlbaLearning (Espagne). Berenice. <http:// albalearning.com/audiolibros/poe/berenice-sp. $\mathrm{html} />$, [Consulta el I5 octubre 2009].

AlbaLearning (Espagne). El retrato oval. <http:// albalearning.com/audiolibros/poe/elretratoovalsp-en.html> [Consulta el I5 octubre 2009].

AlbaLearning (Espagne). La máscara de la muerte roja <http://albalearning.com/audiolibros/poe/ lamascara-sp.html> [Consulta el I5 octubre 2009].

Micó Romero, N. (2002). "El marcador temporal déjā", in Gloria Luque Agulló, Antonio Bueno González \& Gabriel Tejada Molina (dir.), Las lenguas en un mundo global, AESLA: Universidad de Jaén (Servicio de publicaciones). Publicación en CD ROM.

Micó Romero, N. (2005). "Un aspecto del discurso ensayístico francés: los marcadores temporales". Quaderns de Filologia, Io/ 2005, pp. 187-204.

Bosque, I. \& Demonte, V. (2000 [I999]). Gramática descriptiva de la lengua española. Vol I: Sintaxis básica de las clases de palabras. Madrid: Espasa Calpe.
Cuenca, M. J. \& Hilferty, J. (I999). Introducción a la lingüistica cognitiva, Barcelona: Ariel.

Dictionnaire unilingue anglais (20II). Collins English Dictionary. London : Harper Collins Publishers.

Franckel, J. J. (I989). Étude de quelques marqueurs aspectuels du français. Ginebra/Paris: Librairie Droz.

Giordano, R. Berenice [en línea]: poemas y novelas cortas de Edgar Allan Poe. 2005, <http:// poestories.com/read/berenice> [Consulta el I5 octubre 2009].

Giordano, R. The masque of the Red Death [en linea]: poemas y novelas cortas de Edgar Allan Poe. 2005, <http://poestories.com/read/masque> [Consulta el I5 octubre 2009].

Giordano, R. The Oval Portrait [en línea]: poemas y novelas cortas de Edgar Allan Poe. 2005, < http:// poestories.com/read/ovalportrait $>$ [Consulta el I5 octubre 2009].

Kleiber, G. (I990). La sémantique du prototype: catégories et sens lexical, Paris : Presses Universitaires de France.

Langacker, R-W. (I987). Foundations of cognitive grammar, Stanford: Stanford University Press.

Reverbere (Francia). Bérénice [en línea]: obras de libre acceso. 2004, http:/www.inlibroveritas.net/ lire/oeuvre22722.html\#page_io6 [Consulta el I5 octubre 2009].

Reverbere (Francia). Le masque de la mort rouge. [en línea]: obras de libre acceso. 2004, http:// www.inlibroveritas.net/lire/oeuvre 22722. html\#page_I99 [Consulta el I5 octubre 2009].

Reverbere (Francia). Le portrait ovale. [en línea]: obras de libre acceso. 2004, < http://www.inlibroveritas. net/lire/oeuvre22722.html\#page_I99> [Consulta el I5 octubre 2009].

Seco, M. (1999). Diccionario del español actual, Madrid: Grupo Santillana de ediciones, S.A. 